\title{
Karotisstenose: Pillen statt OP?
}

\author{
Chefredakteur Dr. med. Dirk Einecke
}

\section{ASYMPTOMATISCHE KAROTISSTENOSE}

\section{Zu oft invasiv behandelt?}

Die medikamentöse Therapie bei stummen Karotisstenosen ist inzwischen so gut, dass sie bei 95\% der Patienten das Schlaganfallrisiko stärker reduziert als eine Endarterektomie oder Stentimplantation. Als Folge der Verbesserungen in der konservativen Behandlung (Blutdruckkontrolle, Lipidsenkung) sank bei beschwerdefreien Patienten mit Karotisstenosen die Prävalenz von Mikroemboli von 12,6\% vor dem Jahr 2003 auf 3,7\% in der Zeit danach. Patienten ohne Mikroemboli haben ein 1-Jahres-Risiko für einen Schlaganfall von $1 \%$. Dem gegenüber

\section{AKUTTHERAPIE DES SCHLAGANFALLS}

\section{Mehr Zeit für die Lyse}

Bisher beträgt das Zeitfenster gerade einmal drei Stunden: Mehr Zeit soll nicht verstreichen zwischen den ersten Symptomen eines ischämischen Schlaganfalls und dem Beginn einer Lysetherapie. Doch eine Studie unter Leitung von Prof.

Werner Hacke aus Heidelberg zeigt, dass eine Thrombolyse auch noch zu einem späteren Zeitpunkt nützlich sein kann. Eine Behandlung mit Alteplase nach 3 bis

\section{INFEKTIOLOGIE}

\section{Honig besser als Antibiotika?}

Honig in Tee ist ein altes Hausmittel bei Halsentzündungen. Dass er tatsächlich ein potenter Bakterienkiller ist, zeigt eine kanadische Studie. Wurde Honig einer Bakterienlösung zugesetzt, tötete er alle getesteten Bakterienstämme - darunter auch methicillinresistente Staphylococcus aureus. Gegen rasenbildende Bakterien war Honig in 63-91\% der Fälle wirksam und damit viel effektiver als Rifampicin. Solche Bakterienfilme bilden sich z. B. bei chronischer Sinusitis. Spülungen mit Honiglösungen könnten hier hoch wirksam sein. Allerdings: Honig ist nicht gleich Honig. Antibakteriell wirksam war Honig aus Neuseeland und dem Jemen. Kanadischer Honig zeigte keine Wirkung.

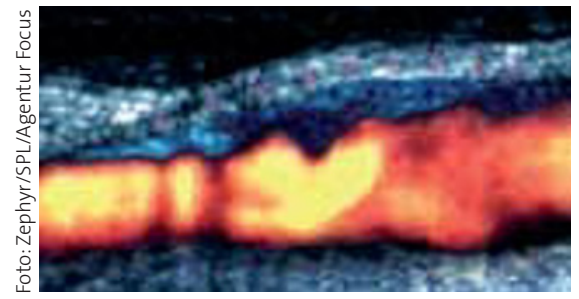

(K)ein Fall für den Gefäßchirurgen?

steht ein $5 \%$ iges Risiko für Schlaganfall oder Tod bei einem interventionellen oder operativen Eingriff. Der Eingriff lohnt sich daher vor allem für die wenigen Patienten mit Mikroemboli; sie haben ein Risiko von $14 \%$ für einen Insult im nächsten Jahr. World Stroke Congress, Wien, 25. September 2008

4,5 Stunden führte zu deutlich besseren Ergebnissen als eine Placebobehandlung. Lediglich intrakranielle Blutungen waren etwas häufiger als unter Placebo. „Mehr Zeit zu haben heißt aber nicht, dass man sich mehr Zeit lassen darf", betonte Hacke. Studien belegen, dass die Ergebnisse der Lyse umso besser sind, je früher damit begonnen wird.

N Engl J Med 2008;359:1317-1329

\section{DICKE ASTHMATIKER}

\section{Steroide weniger wirksam}

Glukokortikoide wirken bei übergewichtigen und adipösen Asthmapatienten um etwa $40 \%$ schwächer als bei normalgewichtigen Patienten. Diese klinische Beobachtung wurde in einer Studie auch in vitro bestätigt. Bei 45 Erwachsenen, von denen 33 unter Asthma litten, zeigte sich bei Asthmapatienten mit erhöhtem Bodymass-Index ein vermindertes Ansprechen auf Dexamethason, gemessen an einer verminderten MPA-1-Expression in Blutzellkulturen. Als klinische Konsequenz empfehlen die Autoren, bei übergewichtigen Asthmatikern, deren Erkrankung mit der bisherigen Behandlung unzureichend kontrolliert ist, die Dosis inhalativer Steroide zu erhöhen.

American Journal of Respiratory and Critical Care Medicine vom 1.10.2008
HORMONERSATZTHERAPIE

\section{Auf das „Wie“ kommt es an}

Ob die Hormonersatztherapie bei menopausalen Frauen mit einem erhöhten kardialen Risiko einhergeht oder nicht, wird weitgehend davon bestimmt, wie die Hormone appliziert werden. Dies ergab eine dänische Beobachtungsstudie an nahezu 700000 gesunden Frauen im Alter zwischen 51 und 69 Jahren, die von 1995 bis 2001 durchgeführt wurde. Insgesamt gab es bei den Hormonanwenderinnen nicht mehr Herzinfarkte als bei nicht behandelten Frauen. Bei jüngeren Frauen (51-54 Jahre) war das Risiko jedoch im Vergleich zu Frauen ohne Hormonersatztherapie um $24 \%$ erhöht. Auch Frauen, die kontinuierlich Östrogen plus Progesteron einnahmen, hatten ein (um $35 \%)$ höheres Infarktrisiko.

Wurden die Hormone zyklisch genommen, bestand hingegen sogar die Tendenz zu einem - im Vergleich zu unbehandelten Frauen - verringerten Infarktrisiko. Bei transdermaler oder vaginaler Applikation der Östrogene lag das Risiko sogar um mehr als ein Drittel niedriger. Eur Heart J 2008, doi:10.1093/eurheartj/ehn408

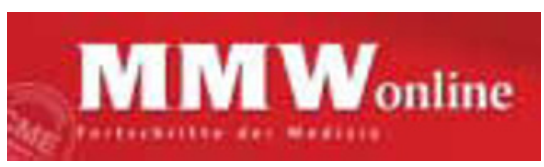

MMW ONLINE

\section{Information auf allen Kanälen}

Auf MMW online gibt es sieben „Kanäle“: Home, Aktuelle Medizin, Fortbildung/ CME, Unternehmen Arztpraxis, Downloadcenter, Archiv und Service. In den kommenden Wochen werden wir Ihnen jeden einzelnen Kanal vorstellen. Diese Woche „Home“, die Startseite von MMW online: Hier finden Sie eine Auswahl der aktuellsten Beiträge sowie Kurznachrichten und Kongressankündigungen. Zudem haben Sie die Möglichkeit, den MMW-Newsletter zu bestellen und sich direkt die CMEFortbildungen der MMW auf CME-Punkt. de abzurufen. Sie können Kontakt zur Redaktion aufnehmen, Ihre Daten verwalten und vieles mehr. 